Leitfaden für die Praxis

\title{
Unklar erhöhte Transaminasen und die klinische Bedeutung der nichtalkoholischen Fettleber
}

\author{
M. Karner, W. Stremmel
}

Innere Medizin IV (Gastroenterologie, Hepatologie, Infektionskrankheiten, Vergiftungen), Medizinische Universitätsklinik, Heidelberg

(Direktor: Prof. Dr. W. Stremmel)

klinikarzt 2004; 33 (7): 220-226

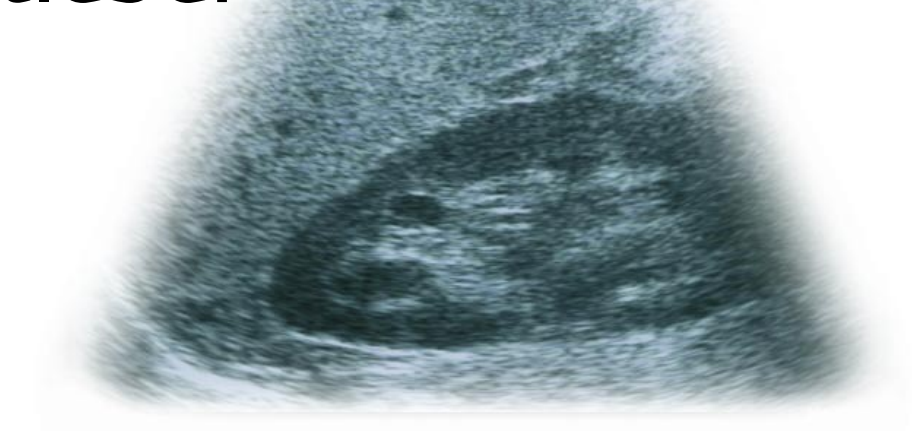

Die Fettleber - ein häufiger Zufallsbefund bei einer bildgebenden Untersuchung - ist mit einer Prävalenz von etwa 20\% die häufigste pathologische Leberveränderung in der westlichen Welt. Ihr pathologischer Charakter ist jedoch noch nicht definitiv geklärt. Zur Entzündung der Fettleber kommt es nach heutigem Verständnis, wenn ihre Schädigung durch hepatotrope Noxen wie zum Beispiel Medikamente, Alkohol, bakterielle Toxine aus der Darmflora, Hyperglykämie oder Hyperlipidämie eine hypothetische Toleranzschwelle der Leber übersteigen („Double-hitHypothese“). Bevor die Diagnose jedoch endgültig festgestellt werden kann, müssen andere Lebererkrankungen differenzialdiagnostisch ausgeschlossen werden. Bestätigt sich die Diagnose „Steatohepatitis“, müssen zur ursächlichen individuellen Therapie die häufig multifaktoriellen Ursachen der Erkrankung abgeklärt werden (z.B. Diabetes: Insulinsensitizer, optimierte Insulintherapie; Adipositas: kalorienreduzierte Diät). Unabhängig von der Pathogenese werden zusätzlich häufig Ursodeoxycholsäure, Betain, Vitamin E und Gemfibrozil eingesetzt obwohl die Effizienz dieser Therapiestrategien nicht oder noch nicht ausreichend durch evidenzbasierte Studien belegt ist.

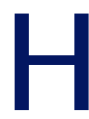
äufig weisen asymptomatische Patienten, die glaubhaft wenig Alkohol trinken, „erhöhte Leberwerte“ im Routinelabor auf. Wenn sich darauf in der Sonografie die Echodichte gegenüber dem Nierenparenchym vermehrt darstellt, liegt die Diagnose der nichtalkoholischen Fettleber (NASH) nahe. Da jedoch andere Lebererkrankungen ebenfalls eine Steatosis verursachen können, müssen diese ausgeschlossen werden.

\section{Abklärung erhöhter \\ Transaminasen}

Hepatitiden verschiedenster Genese führen im Verlauf der Jahre häufig zum fibrotischen Umbau der Leber. Um eine Leberkrankheit rechtzeitig zu erkennen und richtig einzuordnen, hilft die Labordiagnostik, welche hier im Überblick dargestellt ist.

\section{Cholestase}

Ob der Galleabfluss behindert ist, kann man aufgrund einer Erhöhung der Cholestaseparameter alkalische Phosphatase, GGT und konjugiertes Bilirubin feststellen. Bei erhöhten Transaminasen liegt eine Cholangitis-Komponente vor.

Eine intrazelluläre Cholestase findet sich im Rahmen von Hepatitiden jedweder Ätiologie oder medikamenteninduziert durch Interferenz mit Transportvorgängen in Hepatozyten. Bei der obstruktiven Cholestase dagegen führt eine me- chanische Verlegung der Gallengänge zu einem Rückstau der Gallensäuren. Deren toxische Wirkung verursacht eine sekundäre Leberzellschädigung mit einem Anstieg der Transaminasen (cholangitische Komponente). Das sonografische Bild erinnert dann an eine doppelläufige Schrotflinte (Doppelflintenphänomen). In milden Formen der obstruktiven Cholestase können lediglich die alkalische Phosphatase und die Gamma-GT erhöht sein. Sensitiver als die Sonografie ist jedoch die Darstellung der Gallengänge mithilfe einer endoskopisch retrograden Cholangiopankreatikografie (ERCP).

Eine obstruktive Cholestase ist oft Folge von Gallensteinen oder aber von Raumforderungen (maligne oder benigne Tumoren oder Regeneratknoten der zirrhotischen Leber). Aber auch Stenosen der Gallengänge, wie sie bei der primär sklerosierenden Cholangitis (PSC) oder der primär biliären Zirrhose (PBC) vorkommen, können zu Cholestasezeichen führen. Selbst die Fettleber mit Kompression des Parenchyms könnte zur Störung des Galleflusses beitragen.

\section{Virusserologie}

Im Rahmen der Virusserologie sollten Infektionen mit dem Hepatitis-B- sowie dem Hepatitis-C-Virus ausgeschlossen werden (anti-Hbc- 
und Hbs-Ag; anti-HCV), im akuten Fall ist auch an eine Hepatitis A (anti-HAV) zu denken. Ist die Hepatitis-B-Serologie positiv, könnte in seltenen Fällen eine Koinfektion mit Hepatitis-D-Viren vorliegen - nachgewiesen über den anti-HDV-Antikörper. Eher selten ist eine Hepatitis E, die tatsächlich nur nach Auslandsaufenthalten wie zum Beispiel in Indien, China oder Mexiko eine Rolle spielen sollte. Wie die Hepatitis A zeigt diese jedoch nie einen chronischen Verlauf.

Herpesviren können ebenfalls Hepatitiden hervorrufen:

- Ebstein-Barr-Virus (EBV)

- Cytomegalie-Virus (CMV)

- Varizella-Zoster-Virus (VZV).

Diese letzten drei Virushepatitiden sind jedoch deutlich seltener und treffen häufiger Immungeschwächte oder Kinder - bei einer unklaren Hepatitis sollten sie jedoch ausgeschlossen werden.

\section{Hämochromatose}

Die Hämochromatose - eine autosomal rezessiv vererbte EisenSpeicherkrankheit - ist mit einer Prävalenz von etwa 1:400 relativ häufig. Auch hier kann das Labor erste diagnostische Hinweise liefern: Typischerweise sind Ferritin und die Transferrinsättigung mit Eisen im Serum deutlich erhöht, da aufgrund eines Gendefekts im Hämochromatose-Gen HFE die Eisenresorptionsrate auf etwa das Doppelte ansteigt.

Da Ferritin als Akutphasenprotein bei akuten Hepatitiden und anderen Entzündungen erhöht sein kann, kann zur Abklärung ein HFEGentest durchgeführt werden (Bestimmung der C282Y- und H63DMutation). Damit ist eine Hämochromatose zwar zu sichern, bei negativem Ergebnis jedoch nicht mit letzter Sicherheit ausschließen.

\section{Kupfer-Speicherkrankheit}

Wesentlich seltener als die Hämochromatose ist der ebenfalls autosomal rezessiv vererbte Morbus Wilson. Dennoch sollte bei erhöhten Transaminasen die Wilson-Krankheit unbedingt ausgeschlossen werden: Hinweisend für die Diagnose ist die erhöhte Kupferkonzentration im
Sammelurin (über $80 \mu \mathrm{g} / \mathrm{Tag}$ ) und die Verminderung des Kupfer- und des Coeruloplasminspiegels im Serum (Serumkupfer unter $70 \mu \mathrm{g}$ ). Durch die systemische Kupferüberladung, kann es zur Schädigung des Nervensystems und innerer Organe kommen. Fulminante Verläufe sind möglich.

\section{Autoimmunhepatitis}

Ein erhöhter Titer der Immunglobuline und der Nachweis von Autoantikörpern wie zum Beispiel antinukleäre Antikörper (ANA), Leber-Nieren-Mikrosomen-Antikörper („liver kidney microsome“; LKM), Antikörper gegen glatte Muskulatur („smooth muscle antibodies“; SMA) und Antikörper gegen lösliches Leberantigen („soluble liver antibody“; SLA) oder auch von Gliadinund Endomyosin-Antikörper können auf Autoimmunhepatitiden (AIH) hinweisen.

Doch auch wenn solche Antikörper fehlen, kann die Autoimmunhepatitis nicht definitiv ausgeschlossen werden. Zudem können begleitende Autoimmunerkrankungen einen Hinweis auf die AIH-Diagnose geben. Auch die Histologie kann zur Diagnostik herangezogen werden, sie ist aber nicht eindeutig. Schwierig ist die Diagnose von Autoimmunhepatitiden nach Ausbildung einer Leberzirrhose, da - unabhängig von der Ätiologie - die Immunglobuline gegenüber den von der Leber produzierten Proteinen erhöht sein können.

\section{Primär sklerosierende Cholangitis}

Bei der primär sklerosierenden Cholangitis kommt es zu einer idiopathischen, möglicherweise immungetriggerten Sklerosierung der Gallengänge, die mit einer Erhöhung der Cholestaseparameter einhergeht. Die Transaminasen dagegen sind meist nur gering oder nicht erhöht. In etwa 65\% der Fälle sind die P-ANCAs („perinuclear anti-neutrophile cytoplasmic antibodies“) erhöht, jedoch nicht spezifisch. Wegweisend für die Diagnose ist die endoskopisch retrograde Cholangiopankreatikografie (ERCP), welche Kalibersprünge und Stenosen der Gallengänge gut abbildet.

\section{Tab. 1 Prognostisch ungünstige Faktoren}

- Alter über 45 Jahre

- Diabetes mellitus

- Adipositas

- GOT > GPT (positiver de-Ritis-Quotient)

- histologisch „progrediente Form“

- Leberfibrose

Primar biliäre Zirrhose

Ähnlich wie bei der primär sklerosierenden Cholangitis kommt es bei der primär biliären Zirrhose zur Engstellung der Gallengänge. Über einen vermuteten Autoimmunmechanismus sind hier jedoch eher die kleinen Gallengänge betroffen. Die alkalische Phosphatase ist bei diesen Patienten besonders erhöht. Die Diagnose wird durch die Histologie und antimitochondriale Antikörper (AMA) gestützt.

\section{Alpha-1-Antitrypsin-Mangel}

Eine weitere genetische Erkrankung mit einer Häufigkeit von ungefähr 1:2000 ist der Alpha-1-Antitrypsin-Mangel, der aufgrund der erniedrigten Rate von Alpha-1-Antitrypsin ( $\alpha 1-\mathrm{AT})$ im Serum diagnostiziert werden und mit einem obstruktiven Lungenemphysem assoziiert sein kann. Bereits im Kindesalter kann diese Krankheit symptomatisch werden, sie kann aber ebenso asymptomatisch verlaufen und sich erst nach einer zusätzlichen Leberschädigung manifestieren.

\section{Hepatische Porphyrien}

Hepatische Porphyrien haben eine Inzidenz von etwa 1:5000, am häufigsten sind die akute intermittierende Porphyrie und die Porphyria cutanea tarda. Der Nachweis erfolgt über Metabolite aus dem Porphyrinstoffwechsel im Sammelurin.

\section{Transaminasenerhöhung ohne Hepatitis}

Erhöhungen der Transaminasen müssen nicht zwangsläufig mit einer Entzündung der Leber einhergehen. So haben etwa 3\% der Bevölkerung bereits aufgrund der Normwertfestlegung der Transaminasen erhöhte Serumwerte ohne Krankheitswert. Jedoch sind in diesen Fäl- 
Tab. 2 Symptomatische medikamentöse Therapie

\begin{tabular}{lllllr} 
Medikament & $\begin{array}{l}\text { Patienten } \\
\text { (n) }\end{array}$ & $\begin{array}{l}\text { Dauer } \\
\text { (Monate) }\end{array}$ & $\begin{array}{l}\text { Trans- } \\
\text { aminasen } \downarrow\end{array}$ & $\begin{array}{l}\text { histologische } \\
\text { Besserung }\end{array}$ \\
\hline Ursodeoxycholsäure & 24 & 12 & ja & ja & $(1)$ \\
\hline Ursodeoxycholsäure & 126 & 24 & nein & nein & $(10)$ \\
\hline Pioglitazon & 18 & 11 & ja & ja & $(13)$ \\
\hline Clofibrat & 16 & 12 & nein & nein & $(17)$ \\
\hline Gemfibrozil & 46 & 1 & ja & - & $(3)$ \\
Betain / Diethanolamin / Vitamin C & 191 & 2 & ja & ja & $(11)$ \\
\hline N-Acetylcystein & 11 & 3 & ja & - & $(17)$ \\
\hline Vitamin E & 11 & $4-40$ & ja & - & $(8)$
\end{tabular}

len die Werte nur marginal erhöht. Demzufolge weisen natürlich auch 3\% der Patienten mit Fettleber erhöhte Transaminasen auf, obwohl keine Leberentzündung besteht. Aufschluss geben kann in diesen Fällen eine Leberpunktion.

Nach sportlicher Betätigung oder bei einer Myositis kommt es - wenn die Membranstruktur der Myozyten geschädigt wird oder eine Myositis entsteht - neben der Erhöhung der Kreatininkinase(CK)- und der Laktatdehydrogenase(LDH)-Spiegel zum Anstieg von GPT (Glutamat-Pyruvat-Transamniase) und GOT (Glutamat-Oxalacetat-Transaminase).

\section{Medikamentös-toxische Hepatitis}

Zahlreiche Medikamente und Agenzien können entweder über eine Leberzellverfettung oder durch eine direkte Schädigung der Hepatozyten eine Hepatitis verursachen. Dementsprechend schwierig kann die Diagnose sein. Im Prinzip kann sie nur bestätigt werden, indem der Reihe nach alle Medikamente abgesetzt werden. Fallen anschließend die Transaminasen in den Normbereich, ist die Diagnose gesichert. Bei einer medikamentös induzierten Fettleberhepatitis kann sich die Regeneration der Leber jedoch verzögert vollziehen, da sich die Leberschädigung im Sinne eines Circulus vitiosus verselbstständigt hat.

Zu den Medikamenten, die häufig mit einer nichtalkoholischen Fettleber vergesellschaftet sind, zählen zum Beispiel:

- Amiodaron

- Kalziumantagonisten

- Chloroquin
- Methotrexat

- Bloamycin

- Tetrazyklin

- Asparaginase.

Pädiatrische Lebererkrankungen

Es gibt eine Vielzahl pädiatrischer Lebererkrankungen wie das AlagilleSyndrom, die Mukoviszidose, die Wolman-Krankheit oder die zahlreichen Glykogenspeicherkrankheiten (z.B. von-Gierke-Syndrom), zum Teil mit fatalen Verläufen. Die Abklärung einer Hepatitis im Kindesalter Bedarf daher eines Spezialisten.

\section{Alkoholische Leberkrankheit}

Nach der Virushepatitis ist Alkohol die häufigste Ursache für eine Leberentzündung. Neben der Dosis von über $30 \mathrm{~g} /$ Tag für Männer und $20 \mathrm{~g} /$ Tag für Frauen ist vermutlich auch die Art und Konstanz des Abusus für die Leberschädigung verantwortlich: Die Frage nach Karenztagen, Spiegel- oder Quartaltrinkverhalten mag für die Leberschädigung entscheidend sein. Jedoch liegt die Inzidenz einer Leberschädigung selbst bei 20-jährigem Alkoholabusus nur bei zirka $10 \%$.

Vermutlich spielt wie bei vielen Lebererkrankungen auch hier die Anzahl der toxischen Agenzien (siehe „Double-hit-Theorie“, Pathogenese) eine wichtige Rolle. Typischerweise ist bei der alkoholischen Hepatitis die Gamma-GT erhöht, und die GOT höher als die GPT (positiver de-Ritis-Quotient). Auch das Bilirubin kann marignal erhöht sein.

\section{Nichtalkoholische Fettleberhepatitis}

Bei der nichtalkoholischen Steatohepatitis (NASH) sind im Gegen- satz zur nichtalkoholischen Fettleber (NAFL) die Transaminasen erhöht. Oft wird dabei fälschlicherweise von einem übertriebenen Alkoholkonsum ausgegangen. Als Ludwig vor 24 Jahren über die nichtalkoholische Fettleberhepatitis berichtete, wurde er meist belächelt. Zu eng war die Fettleberhepatitis gedanklich mit dem Alkoholabusus assoziiert.

Letztlich ist die NASH eine Ausschlussdiagnose, sie ist wie viele Diagnosen in der Hepatologie häufig suggestiv. Sonografie und Histologie können nicht beweisend, aber aufschlussreich sein, da sich die der nichtalkoholischen Fettleberhepatitis zugrunde liegende Fettleber darstellen lässt. Für die Differenzierung zwischen alkoholischer und nichtalkoholischer Steatohepatitis eignet sich die Histologie jedoch nicht.

Auch gegenüber anderen Leberkrankheiten kann die Histologie meist nur ein Baustein zur Diagnose sein. Beispielsweise verursachen Leberschädigungen wie durch $\mathrm{M}$. Wilson, Hämochromatose oder Hepatitis $C$ oft eine Leberzellverfettung und können so eine vorbestehende Fettleber vortäuschen. Laborchemisch führt bei der nichtalkoholischen Fettleberhepatitis die GPT üblicherweise vor der GOT (negativer de-Ritis-Quotient). Nur im schweren Verlauf bei mitochondrialer Mitbeteiligung kommt es zur Umkehr des Quotienten mit führender GOT.

Das Bilirubin liegt üblicherweise im Normbereich. Die Differenzialdiagnostik der nichtalkoholischen Steatosis hepatis fasst Tabelle $3 \mathrm{zu}-$ sammen. 
Basis ist die nichtalkoholische

Fettleber

Die nichtalkoholische Fettleber ist zumeist eine sonografische Diagnose, da die histologische Sicherung nicht praktikabel und den Patienten meist nicht zuzumuten ist. Streng genommen bezieht sich die Definition der Fettleber jedoch auf den histologischen Befund: In über 50\% der Hepatozyten sind Lipideinlagerungen darzustellen. Obwohl die Diagnose so häufig ist - zirka $20 \%$ der Gesamtbevölkerung haben eine Fettleber -, sind klinische Bedeutung und Prognose weit gehend ungewiss. Überraschenderweise weisen 15-50\% der Patienten keine Risikofaktoren auf.

\section{Die Epidemiologie der Fettleber}

Die Steatosis hepatis ist die häufigste Veränderung der Leber in den westlichen Ländern. Ihre Häufigkeit variiert jedoch von Studie zu Studie und hängt von sozialen und regionalen Unterschieden $a b$, daher sind hier nur grobe Zahlenangaben genannt:
- $\quad 1 / 6$ der gesunden Bevölkerung

- 1/3 der hospitalisierten Patienten

- 1/2 der Alkoholkranken

- $1 / 2$ der Diabetiker

- 3/4 der adipösen Bevölkerung

- $\quad 95 \%$ der adipösen Trinker.

Aufgrund der Zunahme von Adipositas, Diabetes und metabolischem Syndrom gewinnen Fettleber und Fettleberhepatitis immer mehr an Bedeutung. Auch die medikamentös induzierte Steatosis könnte aufgrund der steigenden Verordnungshäufigkeit der Medikamente immer häufiger werden.

\section{Diagnose und klinische \\ Bedeutung}

Häufigster Zufallsbefund in der Sonografie des Erwachsenen ist die erhöhte Echodichte der Leber gegenüber dem Nierenparenchym mit inhomogener Echostruktur - ein so genannter „Leberparenchymschaden vom Steatose-Typ“. Auch im Rahmen von anderen Bildgebungsuntersuchungen, wie dem MRT oder
CT, kommt es oft zu diesem Nebenbefund.

Der tatsächliche pathologische Charakter einer Fettleber ist im Einzelfall jedoch unklar. Liegen gleichzeitig erhöhte Transaminasen vor, muss von einer Entzündung, also einer nichtalkoholischen Steatohepatitis, ausgegangen werden. $\mathrm{Zu}$ bedenken ist jedoch, dass etwa 3\% der Bevölkerung als Normvariante erhöhte Leberwerte aufweisen oder auch dass eine NASH oder eine Fettleberzirrhose ohne laborchemisches Korrelat entstehen können. Klarheit darüber liefert der histologische Befund. Über 50\% der Patienten mit einer Steatosis hepatis zeigen zum Zeitpunkt der Diagnose fibrotische Zeichen und bis zu 15\% Zeichen des zirrhotischen Leberumbaus.

\section{Potenzielle Ursachen}

Viele mögliche Ursachen der Fettleber lassen sich bereits aus der Epidemiologie ablesen. Ein großer Teil der Patienten mit Fettleber (15-50\%) hat jedoch keinerlei Risikofaktoren für eine Fettleber, nur

\section{Tab. 3 Differenzialdiagnostik der nichtalkoholischen Steatohepatitis}

\begin{tabular}{|c|c|c|}
\hline \multirow{3}{*}{$\begin{array}{l}\text { infektiöse } \\
\text { Ursachen }\end{array}$} & Hepatitis A-C & Anti-HAV, HBs-Ag \& Anti-HBc (ggf. Anti-HBs), Anti-HCV \\
\hline & Hepatitis D bzw. E & $\begin{array}{l}\text { Serologie falls Infektion möglich (HBV-Infektion bzw. Auslands- } \\
\text { aufenthalte in Risikogebieten) }\end{array}$ \\
\hline & CMV, VZV, EBV & Virusserologie \\
\hline \multirow{5}{*}{$\begin{array}{l}\text { genetische und } \\
\text { metabolische } \\
\text { Ursachen }\end{array}$} & Hämochromatose & Ferritin, Transferrinsättigung, ggf. HFE-Test \\
\hline & M. Wilson & Coeruloplasmin, Kupfer im Sammelurin, ggf. Histologie \\
\hline & Porphyrie & Porphyrine im Sammelurin (abgedunkelt sammeln) \\
\hline & pädiatrische Ursachen & Vorstellung beim Spezialisten \\
\hline & Alpha-1-AT-Mangel & Alpha-1-Antitrypsin ist vermindert \\
\hline \multirow[t]{6}{*}{ Cholestase } & Tumor & Cholestaseparameter (AP, GGT, Bilirubin) \\
\hline & & Sonografie, ggf. Tumormarker (z.B. CEA, CA19-9, AFP) \\
\hline & & ERCP, ggf. weitere Bildgebung, Feinnadelpunktion \\
\hline & Cholelithiasis & Sonografie, ERCP oder andere Bildgebung \\
\hline & PSC & PANCA, ERCP \\
\hline & PBC & AMA, Histologie \\
\hline $\begin{array}{l}\text { Autoimmun- } \\
\text { hepatitis }\end{array}$ & & $\begin{array}{l}\text { Immunglobulinfraktion, Anti-SLA, ANA, ANCA, Anti-LKM, glatte } \\
\text { Muskulatur Antikörper, Gliadin- und endomysiale Antikörper usw. }\end{array}$ \\
\hline medikamentös & & Kontrolle der Medikation und probatorisches Absetzen bzw. \\
\hline induziert & & Pausieren \\
\hline alkoholinduziert & & Anamnese, meist GOT > GPT und GGT erhöht, CDT \\
\hline \multirow[t]{6}{*}{ NASH } & & GPT > GOT, glaubhaft kein übertriebener Alkoholkonsum \\
\hline & $\begin{array}{l}\text { bakterielle Darm- } \\
\text { überwucherung }\end{array}$ & klinische Hinweise $\rightarrow$ Atemtest und Stuhluntersuchung \\
\hline & Diabetes & Glukoseintoleranztest, $\mathrm{HbA}_{1 \mathrm{c}}$ \\
\hline & Adipositas & Habitus, BMI > 25 \\
\hline & Kachexie, Anorexie & Habitus, BMI < 20 \\
\hline & Hyperlipidämie & Triglyzeride, Cholesterin, VLDL \\
\hline
\end{tabular}




\section{Abb. 1 Sonografie einer Fettleber}

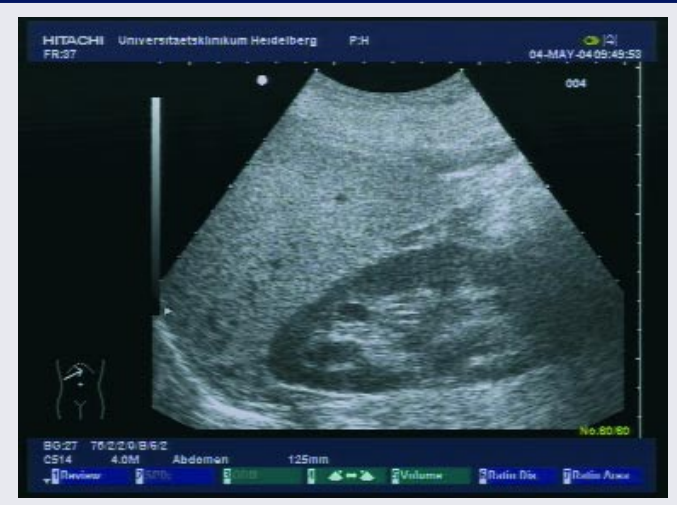

Das Leberparenchym ist gegenüber dem Nierenparenchym deutlich echoreicher, hier ausgeprägt im Sinne einer so genannten „weißen Leber“

teilweise lassen sich subklinische Krankheitsbilder wie ein latenter Diabetes oder eine subtile Fettstoffwechselstörung als Ursache finden. Oft bleibt die Fettleber trotz ausgiebiger Diagnostik unklar. Ein Spezialfall sind Patienten mit erniedrigten Triglyzeriden. Hier kann es sich um einen Apolipoproteinmangel Typ B (Abeta- oder Hypobetalipoproteinämie) handeln, bei dem Triglyzeride nicht mehr aus dem Hepatozyten ausgeschleust werden können und sich ablagern.

Patienten im höheren Alter neigen bei gleichem Gewicht vermehrt zur Leberverfettung. Der Grund ist nicht bekannt, eventuell könnten sich abzeichnende Stoffwechselstörungen wie eine Insulinresistenz dafür verantwortlich sein. Auch Patienten mit chronischer Hepatitis C,
Morbus Wilson oder Träger der Hämochromatose entwickeln häufig eine Leberverfettung. Hypothetisch werden oxidativer Stress und intrazelluläre Dysfunktion der Leber verantwortlich gemacht

Interessanterweise kann eine Insulinresistenz zur Fettleber und eine Fettleberentzündung zur Insulinresistenz führen. Es könnte also sein, dass sich beide Entitäten gegenseitig in ihrer Entstehung unterhalten.

Patienten mit Anorexia nervosa, Mangelernährung oder Kachexie, konsumierenden Erkrankungen oder Patienten unter strenger Diät (Nulldiät, „gastric banding“) können paradoxerweise eine Fettleber und in der Folge auch eine nichtalkoholische Steatohepatitis entwickeln. Dabei fluten in der katabolen Stoffwechsellage Energieträger wie Lipide oder Ketonkörper an, die von der Leber nicht mehr verstoffwechselt werden können. Häufig besteht gleichzeitig ein Proteinmangel, der zu einer verminderten Produktion von für den Leberstoffwechsel essenziellen Enzymen und Transportern führen kann. Konsekutiv können bei hepatozellulärer Dysfunktion Energieträger nicht mehr regelgerecht aus der Leberzelle geschleust werden.

\section{Pathophysiologie}

Trotz aller Theorien, Diskussionen und molekularer Spekulationen ist die Entstehung der Fettleber unklar. Auch die Double- bzw. Multihit-Theorie, die zwar weit gehend anerkannt wird, bleibt eine Hypo-

\section{Abb. 2 Multifaktorielle Genese der Fettleber}

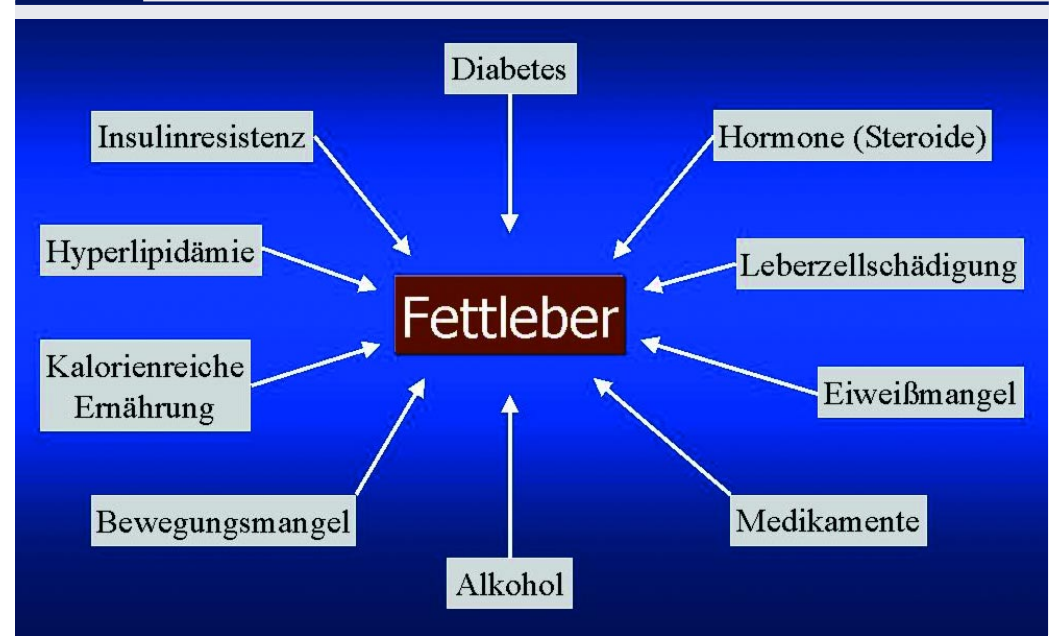

these. Mit einem „hit“ ist ein leberschädigendes Agens gemeint. Der „first hit" soll die Entstehung der Fettleber in der Regel auf der Grundlage eines Ungleichgewichts zwischen Kalorienzufuhr und Kalorienverbrauch im Hepatozyten bedingen (z.B. bei Hyperglykämie, Fettstoffwechselstörungen, Bewegungsmangel oder Adipositas). Überflüssige Energieträger werden nicht ausgeschleust, sondern zu Triglyzeriden synthetisiert und als intrazelluläre Fetttröpfchen eingelagert.

Erst wenn ein zweites hepatotropes Agens - der "second hit“ - auf die Leber einwirkt, kommt es nach dieser Theorie zur Entzündung der Leber. Im Rahmen dieser Entzündung folgt eine Dysfunktion der Leberzellen, und weitere Stoffwechselvorgänge, Transportmechanismen und der Kalorienverbrauch der Leber werden gestört. Nachfolgend lagern die Hepatozyten vermehrt Lipide ein, und der Kreis schließt sich zum Circulus vitiosus.

Eine Schlüsselrolle der Pathogenese der NASH wird einem Ungleichgewicht der Darmflora zugeschrieben. Dafür werden neben Malabsorption und -assimilation auch intestinale Dysmotilitäten, wie sie bei Diabetes und Adipositas vorkommen, verantwortlich gemacht.

Die Produktion toxischer Substanzen im Darm - wie zum Beispiel Lipopolysaccharide, Ethanol und Ammoniak, die über die Pfortader in den Hepatozyten gelangen und in der verfetteten und vorgeschädigten Leber nicht mehr effektiv abgebaut werden können - soll eine toxische Schädigung und Entzündung verursachen (,liver-lumen interaction“). Da zudem die mitochondrialen Reduktionssysteme, die dem Schutz vor oxidativem Stress dienen, erschöpft sind, stünde die Leberzelle solchen toxischen Agenzien mit nur vermindertem Schutz gegenüber. Durch die toxische Zellschädigung sollen TNF- $\alpha$ und andere Zytokine freigesetzt werden, was wiederum konsekutiv zur Migration von Entzündungszellen in die Leber und über einen zweiten Circulus vitiosus zu weiterem Zellzerfall beiträgt.

Es ist gut belegt, dass sich nach operativen Eingriffen am Darm eine 
nichtalkoholische Fettleberhepatitis entwickeln kann. Die Ursache scheint neben der katabolen Stoffwechsellage eine bakterielle Überwucherung des Darms zu sein, da eine antibiotische Therapie als wirksam beschrieben wurde. In den 70er Jahren waren einige Fälle einer fulminanten Fettleberhepatitis nach Anastomose zwischen Jejunum und Ileum (J-I-Bypass) aufgetreten zum Teil mit letalem Ausgang.

\section{Therapieoptionen}

Die Ursachen der Leberverfettung sind mannigfaltig, die Behandlung der Fettleber primär ursächlich, die Indikation zur Behandlung der Fettleber ohne Entzündung meist unklar. Aufgrund der Häufigkeit und der meist völlig benignen Entität kann jedoch nicht jede Fettleber behandelt werden. In der Praxis wird sie häufig nur dann therapiert, wenn erhöhte Transaminasen vorliegen. Jedoch kann sich eine nichtalkoholische Steatohepatitis und eine Fettleberzirrhose in seltenen Fällen laborchemisch unbemerkt entwickeln. Die richtige Entscheidung liegt also in der Hand des Mediziners.

Als Basis für eine Therapieentscheidung können einige prognostische Faktoren für die Entwicklung einer Leberzirrhose auf der Grundlage einer Fettleber dienen (Tab. 1). Im Zweifelsfall kann der histologische Befund weiterhelfen, der (den Grad der) Entzündung und Fibrose darstellen kann. Jedoch sollte auch bei Fehlen jeglicher Risikofaktoren eine weiterführende Diagnostik betrieben werden, um einer abwendbaren, langjährigen Leberschädigung entgegenzuwirken.

Sollte sich die Diagnose der Fettleberhepatitis bestätigen, muss die Pathogenese der Leberverfettung aufgeklärt werden, um eine ursächliche Therapie $\mathrm{zu}$ ermöglichen. Dementsprechend erhalten Diabetiker Insulinsensitizer (z.B. Metformin, Pioglitazon) oder eine optimierte Insulintherapie. Im Falle einer Hypercholesterinämie werden Cholesterinsynthese-Hemmer eingesetzt bzw. eine Ernährungsumstellung auf fettarme Kost empfohlen, bei Adipositas eine Be- wegungstherapie und eine vorsichtige Diät (nur 10\% Gewichtsabbau pro Jahr) induziert. Auf Alkohol sollten die Patienten zunächst verzichten.

Unabhängig von der Pathogenese werden Ursodeoxycholsäure (UDCA), Vitamin E und Gemfibrozil zur Behandlung verwendet. Hoffnungen wurden insbesondere auf die Therapie mit Ursodeoxycholsäure gesetzt: Die Hepatozyten nehmen diese Substanz als überschüssige Gallensäure auf und müssen sie über einen ATP-abhängigen Transporter sezernieren. Dabei kommt es zum intrazellulären Energieverbrauch, was die überschüssigen Energiereserven des Hepatozyten angreift. Eine aktuelle Studie (10) konnte aber entgegen der vorherigen einarmigen Pilotstudien $(4,6,7)$ keinen Vorteil gegenüber Plazebo nachweisen.

Auch die übrigen klinischen Studien zur medikamentösen Therapie der nichtalkoholischen Steatohepatitis entsprechen nicht den heutigen Kriterien der evidenzbasierten Medizin. Die Studien weisen oft eine geringe Fallzahl auf, und sind einarmig oder nur einfach verblindet. Der primäre Endpunkt der Studien ist häufig nicht valide gewählt, histologische Kriterien fehlen oft. Hinweise für eine Wirkung wurden auch für Acetylcystein und Troglitazon beschrieben, der endgültige Wirkungsnachweis steht jedoch aus. Eine große randomisierte, plazebokontrollierte Doppelblindstudie konnte jedoch eine Wirksamkeit der Kombination von Betain-Glucuronat, Detholamin-Glucurronat und Nikotinamid-Ascorbat nachweisen (11).

Aufgrund der Hypothese der Leber-Darm-Interaktion werden auch probiotische Therapien diskutiert (14). Kosten und Risiken der Therapie sind gering. Doch auch für diese Therapieoption gilt: Bislang ist die Wirkung nicht ausreichend untersucht.

Leidet der Patient alleine an einer Fettleber, ist die Indikation zur Therapie - wie bereits erläutert - noch unklar. Die Patienten empfinden die Leberverfettung jedoch häufig als unphysiologisch und sind daher nur schwer zu diätetischen Maßnahmen $\mathrm{zu}$ motivieren. Abhängig vom $\mathrm{Pa}-$ tienten kann geraten werden, Gewicht zu reduzieren, die Ernährung umzustellen, Ausdauerübungen zu betreiben (wie Schwimmen, Joggen oder einfaches Spazieren gehen, z.B. eine halbe bis eine Stunde pro Tag) und den Alkoholkonsum weit gehend zu reduzieren. Bei Risikopatienten sollte eine medikamentöse Begleittherapie erwogen werden.

Unbehandelt hat die nichtalkoholische Steatohepatitis eine vergleichbare Prognose wie die chronische Hepatitis $C$, ein Progress zur Leberzirrhose mit potenziell letalem Ausgang ist möglich. Andererseits finden sich auch benigne Verläufe ohne Leberfibrose. Möglicherweise ist dies auf die unterschiedlichen pathogenetischen Entitäten zurückzuführen. Im Zweifelsfall würden wir jedoch aufgrund der unklaren Prognose eher zur Behandlung (ursächlich und medikamentös) raten.

\section{A Clinical Approach - \\ Elevated Aminotransferases and the Relevance of the Non-alcoholic Fatty Liver Disease}

The fatty liver is - with a prevalence of over $20 \%$ - the most frequent liver disorder in the western world. The hepatic steatosis progresses to an inflammation if a certain quantity of liver toxic agents overwhelm a hypothetical tolerance threshold of the liver ("double-/multi-hit theory"). To confirm the diagnosis of the nonalcoholic steatohepatitis - NASH, other liver diseases have to be excluded. Therapy is primarily based on the patients' individual cause of the disorder: Obesity is treated with a calorie-reduced diet; diabetes with insulin-sensitizern and so on. Other non-specific treatments as ursodeoxycholic acid, vitamin $E$, betaine and gemfibrozile have been used for the therapy of NASH, although efficacy has not yet been proven sufficiently.

\section{Key Words}

nonalcoholic steatohepatitis (NASH)

- nonalcoholic fatty liver disease

(NAFL) - liver inflammation aminotransferases 


\section{Literatur}

1. Abdelmalek M, Angulo P, Jorgensen RA et al. Beatine for patient with non-alcoholic steatohepatitis: a promising new agent. Gastroenterol 2000, 118: A1444

2. Alba LM, Lindor K. Review article: non-alcoholic fatty liver disease. Aliment Pharmacol Ther 2003; 17: 977-986

3. Basaranoglu M. A controlled trial of gemfibrozil in the treatment of patients with nonalcoholic steatohepatitis. J Hepatol 1999; 31: 384

4. Cerani $R$ et al. Effect of ursodeoxic acid plus diet in patients with non-alcoholic steatohepatitis. Hepatology 1998; 28: 386A

5. Gülbahar $\mathrm{O}$ et al. Treatment of non-alcoholic steatohepatitis with $\mathrm{N}$-acetylcysteine. Gastroenterol 2000; 118: A1444

6. Guma G et al. Ursodeoxicholic acid in the treatment of nonalcoholic steatohepatitis: results of a prospective clinical controlled trial. Hepatology 1997; 26: 387A

7. Laurin J, Lindor KD, Crippin JS et al. Ursodeoxycholic acid or clofibrate in the treatment of nonalcoholic-induced steatohepatitis: a pilot study. Hepatology 1996; 23: 1464-1467

8. Lavine JE et al. Vitamin E treatment of nonalcoholic-induced steatohepatitis in children: a pilot study. J Pediatrics 2000; 136: 734-738

9. Leuschner U. Fettleber und Fettleberhepatitis - NASH und ASH. Bremen: Uni-Med Verlag AG, 2003

10. Lindor KD, Kowdley KV, Heathcote EJ et al. Ursodeoxycholic acid for treatment of nonalcoholic steatohepatitis: results of a randomized trial. Hepatology 2004; 39: 770-778

11. Miglio F, Rovati LC, Santoro A et al. Efficacy and safety of oral betaine glucuronate in nonalcoholic steatohepatitis. Arzneimittelforschung 2000; 50 (8): 722-727

12. Neuschwander-Tetri BA, Caldwell SH. Nonalcoholic steatohepatitis: Summary of an AASLD single topic conference. Hepatology 2003; 37 (5): 1202-1219

13. Promrat K, Lutchman G, Uwaifo Gl et al. A pilot study of pioglitazone treatment for nonalcoholic steatohepatitis. Hepatology 2004; 39 : 188-196

14. Solga SF, Diel AM. Non-alcoholic fatty liver disease: lumen-liver interactions and possible role for probiotics. J Hep 2003; 38: 681-687

15. Stremmel W, Blechacz B, Hermann T et al. Die Therapie der alkoholischen und nichtalkoholischen Steatosis hepatis. Der Internist 2001; 42: 1641-1650

16. Te Sligte K, Bourass I, Sels JP et al. Non-alcoholic steatohepatitis: review of a growing medical problem. Europ Jour Int Med 2004; 15: 10-21 17. Uygun A, Kadayifci A, Isik AT et al. Metformin in the treatment of patients with non-alcoholic steatohepatitis. Aliment Pharmacol Ther 2004; 19: 537-544

\section{Anschrift für die Verfasser Dr. Max Karner Abteilung für Innere Medizin IV - Gastroenterologie Medizinische Universitätsklinik Im Neuenheimer Feld 410 69120 Heidelberg}

Georg Thieme Verlag

Rüdigerstraße 14

70469 Stuttgart

Telefon (0711) 8931-0

Telefax (0711) 8931-2 98

Verlag und Copyright:

Georg Thieme Verlag, Stuttgart

Managing Editors:

Adolf Grünert, Ulm

Winfried Hardinghaus, Osnabrück

Burckart Stegemann, Hagen

Achim Weizel, Mannheim

\section{Wissenschaftliche Beiräte:}

Anästhesiologie: Reiner Dölp, Fulda; Chirurgie: Rainer Engemann, Aschaffenburg; Karl-Josep Paquet, Bad Kissingen; Dermatologie: Hans F. Merk, Aachen; Endokrinologie: Klaus-Henning Usadel, Frankfurt; Ernährung: Adolf Grünert, Ulm; Gastroenterologie: Gerald Holtmann, Essen; Gynäkologie/Geburtshilfe: Matthias W. Beckmann, Erlangen; Herz- und Thoraxchirurgie: Bruno Reichart, München; Hämatologie: Hermann Heimpel, Ulm; HNO: Wolfgang Stoll, Münster: Immunparmako: Wlich Klebergünster; Immunpharmakologie: Ulrich Kleeberg, Berlin; Innere Medizin: Wolfram Domschke, Münster; Eckhart G. Hahn, Erlangen; Intensivmedizin: Jürgen E. Schmitz, Wiesbaden; Kardiologie: Berndt Lüderitz, Bonn; Thomas Meinertz, Hamburg; Klinikhygiene: Franz Daschner, Freiburg; Klinische Chemie: Klaus Dörner, Kiel; Klinisch Pathologie: Paul Hermanek, Erlangen; Klinische Pharmakologie: Martin Wehling, Mannheim; Med. Statistik und Dokumentation: Wilhelm Gaus, Ulm; Mund-Kiefer-Gesichts-Chirurgie: Rainer Schmelzeisen, Freiburg; Nephrologie: Konrad Andrassy, Heidelberg; Neurologie: Eberhard Schneider. Hamburg: Onkologie: Rainer Souchon, Scheid Clemens Uninger, Freiburg; Ophthan, Hagen; Clemer. logie: Gerhard K. Lang, Ulm; Orthopädie: Wolfgang Pförringer, München; Pädiatrie: Friedrich C. Sitzmann, Homburg/Saar; Palliativmedizin: Eberhard Klaschik, Bonn; Pulmologie: Gerhard Schultze-Werninghaus, Bochum; Psychiatrie: Hans-Jürgen Möller, München ; Psychosomatik: Ernst Petzold, Aachen; Qualitätsmanagement: Behrend Behrends, Hamburg; Radiologie: Ingolf P. Arlart, Stuttgart; Strahlentherapie: Michael Wannenmacher, Heidelberg; Thorax- und Gefäßchirurgie: Ludger Sunder-Plassmann, Ulm; Traumatologie: Lothar Kinzl, Ulm; Urologie: Lothar Hertle, Münste

\section{Manuskriptrichtlinien:}

Es werden nur unveröffentlichte Manuskripte angenommen, die auch nicht gleichzeitig an anderer Stele zur Veröffentlichung eingereicht sein dürfen. Autorenrichtlinien können beim Verlag angefordert werden. Für unaufgefordert eingesandte Manuskripte wird keine Haftung übernommen. Der Verla geht davon aus, dass der Autor für alle zur Publikation eingereichten Abbildungen das Veröffentlichungs chungsrecht hat. Der Verlag behält sich vor, Leserbriefe gekürzt wiederzugeben. Die eingereichten Arbeiten für Editorial, Schwerpunkt und Übersich werden einem peer review der Schriftleitung unterzogen. Der Inhalt der übrigen Rubriken und der Supplements liegt in der Verantwortung der Redaktion. Copyright 2004:

Mit dem Abdruck des Beitrages erwirbt der Verlag das alleinige und ausschließliche Recht für die Veröffentlichung in sämtlichen Publikumsmedien sowie Übersetzungen in fremde Sprachen. Nachdruck, fotomechanische Wiedergabe und Speicherung in fotomechanische Wiedergabe und Speicherung in Datenverarbeitungsanlagen, auch auszugsweise, nur nach schriftlicher Genehmigung des Verlags. Die Abbildungen in den Beiträgen stammen, wenn im
Inhaltsverzeichnis (s. o.) nicht anders vermerkt, von den jeweiligen Autoren.

Redakteurin:

Stephanie Schikora, Telefon (0711) 89 31-416 E-Mail: stephanie.schikora@thieme.de Redaktionsleitung:

Günther Buck, Telefon (0711) 89 31-4 40

Redaktionsassistentin:

Sabine Bischoff, Telefon (0711) 89 31-5 51 Telefax (0711) 89 31-322

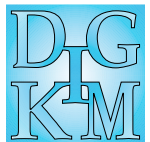

Organ de

Deutschen Gesellschaft

für Interdisziplinäre

Klinische Medizin

Telefon (07 11) 8931-416 Telefax (07 11) 89 31-322
Internet-Adresse:

http://www.thieme.de; http://www.klinikarzt.info

Verantwortlich für den Anzeigenteil:

pharmedia, Anzeigen- und Verlagsservice $\mathrm{GmbH}$

Anzeigenleitung:

Greta Weller, Telefon (0711) 89 31-304

E-Mail: greta.weller@pharmedia.de

Zeitschriftenvertrieb:

Telefon (0711) 89 31-321

Zurzeit gilt die Anzeigenpreisliste vom 1.10.2003

Herstellung:

Werner Schulz, Telefon (0711) 89 31-331

Satz, Grafik, Layout:

Wolfgang Eckl, Werner Schulz, Karl-Heinz Zobel

Druck: Konradin Druck GmbH

70771 Leinfelden-Echterdingen

33. Jahrgang

Druckauflage: 23000 Exemplare;

Verbreitete Auflage: 22100 Exemplare,

(It. IVW 2/96)

Erscheinungsweise: monatlich

Bestellungen:

Über den Buchhandel oder direkt beim Verlag.

Bankverbindung:

Deutsche Bank Stuttgart,

BLZ 60070070 , Kto.-Nr. 1420017

Landesbank Baden-Württemberg

BLZ 600 50101, Kto.-Nr. 2055723

Postgiro Stuttgart

BLZ 60010070 , Kto-Nr. 45000-705

Bezugsbedingungen:

Einzelheft $€ 11,-$ inkl. Porto.

Jahresabonnement $€ 88$,- inkl. MwSt. und Porto. Für Medizinstudenten gegen Nachweis und Bankeinzug $€ 43,50$ inkl. MwSt. und Porto. Ausland zuzügl. Porto $€ 18,80$ (Europa) bzw. 49,80 (Airlift) (cash with order).

Für die Mitglieder der DGIKM ist der Bezug der Zeitschrift im Mitgliedsbeitrag enthalten. De Abonnementpreis umfasst 12 Kalendermonate (Mindestlaufzeit). Abonnements laufen weiter wenn nicht 3 Monate vor Jahresfrist eine Abbestellung beim Verlag vorliegt. Bei Nichtbelieferung im Falle höherer Gewalt, bei Störungen des Betriebsfriedens, Arbeitskampf (Streik, Aussperrung) bestehen keine Ansprüche gegen den Verlag.

M-MED Mitglied der Arbeitsgemeinschaft Leseranalyse medizinischer Zeitschriften e.V.

Regularly listed in EMBASE/ Excerpta Medica

Hinweis: Wie jede Wissenschaft ist die Medizin ständigen Entwicklungen unterworfen. Forschung und klinische Erfahrung erweitern unsere Erkenntnis, insbesondere was Behandlung, medikamentöse Therapie sowie Diagnostik (Laborwerte etc.) anbelangt. Soweit in dieser Zeitschrift Dosierungen, Applikationen oder Laborwerte erwähnt werden, darf der Leser zwar darauf vertrauen, dass Autoren, Herausgeber und Verlag große Sorgfalt darauf verwandt haben, dass diese Angaben dem Wissensstand bei Fertigstellung entsprechen. Fü Angaben über Dosierungsanweisungen, Applikationsformen und Laborwerte kann vom Verlag jedoch keine Gewähr übernommen werden. Jede Benutzer ist angehalten, durch sorgfältige Prüfung der Beipackzettel der verwendeten Präparate und ggf. nach Konsultation eines Spezialisten festzustellen, ob die dort gegebene Empfehlung für Dosierungen oder die Beachtung von Kontraindikationen gegenüber der Angabe in dieser Zeitschrift abweicht. Eine solche Prüfung ist besonders wichtig bei selten verwendeten Präparaten oder solchen, die neu auf den Markt gebracht worden sind. Jede Dosierung oder Applikation erfolgt auf eigene Gefahr des Benutzers. Laborwerte müssen immer auf Ihre Plausibilität geprüft werden und sind abhängig vom jeweiligen Testgerät bzw. Testkit. Autoren und Verlag appellieren an jeden Benutzer, ihm auffallende Ungenauigkeiten dem Verlag mitzuteilen. 